\title{
Prótesis Parcial Removible con Extensión Distal y Apoyo sobre Implantes, Comportamiento y Consideraciones Clínicas
}

\author{
Removable Partial Denture with Distal Extension and \\ Implant Support, Behavior and Clinical Consideration
}

\author{
María José Arellano-Valeria*; Denise Olivares-Keller* \& Marco Antonio Flores**
}

ARELLANO-VALERIA, M. J.; OLIVARES-KELLER, D. \& FLORES, M. A. Prótesis parcial removible con extensión distal y apoyo sobre implantes, comportamiento y consideraciones clínicas. Int. J. Odontostomat., 8(3):419-424, 2014.

RESUMEN: La rehabilitación de los extremos libres, en mandíbula uni o bilaterales, suponen un gran reto para el odontólogo, siendo un problema a resolver en la práctica clínica habitual. Tales casos suelen ser resueltos mediante prótesis parcial removible, las cuales deben soportar fuerzas horizontales y de torsión, con efectos adversos durante la función, al producir fuerzas terminales de torque contra pilares y tejidos blandos principalmente por la diferente resiliencia de estas estructuras afectando así la retención, estabilidad y el soporte de la prótesis dental. En el último tiempo se han reportado prótesis parcial removible mandibular en combinación con implante en extremo libre distal como una alternativa a las tradicionales prótesis parcial removible mucodentosoportada y a las prótesis fijas implantosoportadas para el manejo de la desdentición parcial en extremo libre; sin embargo, los reportes publicados en la literatura sobre esta alternativa es limitada respecto a la funcionalidad y el pronóstico. Este artículo presenta una revisión sistematizada de la literatura cuyo objetivo fue identificar y sintetizar la mejor evidencia disponible respecto a las consideraciones y comportamiento clínico de esta modalidad de tratamiento en desdentados de la clase I y II de Kennedy mandibular.

PALABRAS CLAVE: prótesis parcial removible implantosoportada, prótesis parcial removible sobre implantes, extremo libre.

\section{INTRODUCCIÓN}

La rehabilitación de los extremos libres en mandíbula uni o bilaterales suponen un gran reto para el odontólogo. Tales casos suelen ser resueltos mediante prótesis parcial removible (PPR), las cuales deben soportar fuerzas horizontales y de torsión, con efectos adversos durante la función normal y en las actividades parafuncionales (Turkyilmaz et al., 2009). Los movimientos de rotación de la PPR con extremo libre, debido a la diferentes resiliencias de las estructruras involucradas (diente y mucosa del reborde alveolar distal) (Budtz-Jorgensen, 1999) pueden producir fuerzas terminales de torque contra pilares y tejidos blandos (mucosa, periodonto de los pilares y reborde alveolar del extremo libre) afectando así la retención, estabilidad y soporte de la PPR (de Freitas et al., 2012; Ohkubo et al., 2008).
En el último tiempo se han reportado PPR mandibular en combinación con implante en extremo libre distal (PPRI) como una alternativa a las tradicionales PPR mucodentosoportada y a las prótesis fijas implantosoportadas, para el manejo de la desdentición parcial con extremo libre. Sin embargo, esta alternativa tiene limitaciones respecto a la funcionalidad y pronóstico ya sea a nivel protésico y/o de los implantes, siendo limitada la literatura sobre estos aspectos.

El objetivo de esta revisión sistemática fue identificar y sintetizar la mejor evidencia disponible respecto a las consideraciones y comportamiento clínico de esta modalidad de tratamiento en la extensión distal del desdentamiento parcial mandibular (Clase I o II de Kennedy), en base a la literatura actual.

\footnotetext{
* Estudiante, Escuela de Odontología, Facultad de Odontología, Universidad de La Frontera, Temuco, Chile.

* Departamento de Odontología Integral Adultos, Facultad de Odontología, Universidad de La Frontera, Temuco, Chile.
} 


\section{MATERIAL Y MÉTODO}

Se presenta una revisión narrativa basada en una búsqueda sistemática de la literatura publicada sobre PPR asistida por implantes dentales en extremo libre, entre el 01 de enero de 2003 y el 15 de octubre de 2013, abordando las dimensiones del comportamiento clínico, en cuanto a su efectividad y pronóstico, buscando respuesta a la pregunta ¿qué evidencia actual existe del comportamiento clínico de las PPRI y cuáles son las consideraciones para el manejo de los extremos libres mandibulares con esta modalidad de tratamiento? Para responder estas interrogantes se plantean los siguientes criterios de selección de la información (Fig. 1.).

Se incluyó literatura publicada entre el 01 de enero de 2003 y el 15 de octubre de 2013, siendo la unidad de análisis; seres humanos, de ambos sexos, de entre 20 y 70 años además de pruebas in vitro. Tipo de estudios: metaanálisis, revisiones sistemáticas, estudios comparativos y ensayos clínicos controlados, con artículos completos disponibles, sin límite de idioma, que consideren el tratamiento con PPRI de pacientes con extremo libre mandibular uni o bilateral (Clase I ó Il de Kennedy).
Se excluyeron artículos que no detallan la metodología empleada, ensayos clínicos que consideren pacientes con patologías sistémicas no controladas, tamaño de muestra menores a 5 sujetos, tiempo de seguimiento menor a 4 semanas, uso de mas de un implante por extremo libre o en posición del canino o diente anterior.

La información fue obtenida mediante buscadores de literatura médica, utilizándose las siguientes bases de datos: The Cochrane Central Register of Controlled Trials; Medline (a través del motor de búsqueda PubMED), Dentistry \& Oral Sciences Source y Scientific Electronic Library Online (SciELO).

\section{RESULTADOS}

Problemática Clínica del Extremo Libre. La principal problemática del tratamiento con PPR de extremos libre, es la presencia de diferente respuesta visco elástica dado por el pilar dentario hacia mesial de la base protésica y el tejido blando hacia distal; donde la fuerza oclusal tiende a hacer que la base se mueva en mayor magnitud, ya que los dientes artificiales pos-

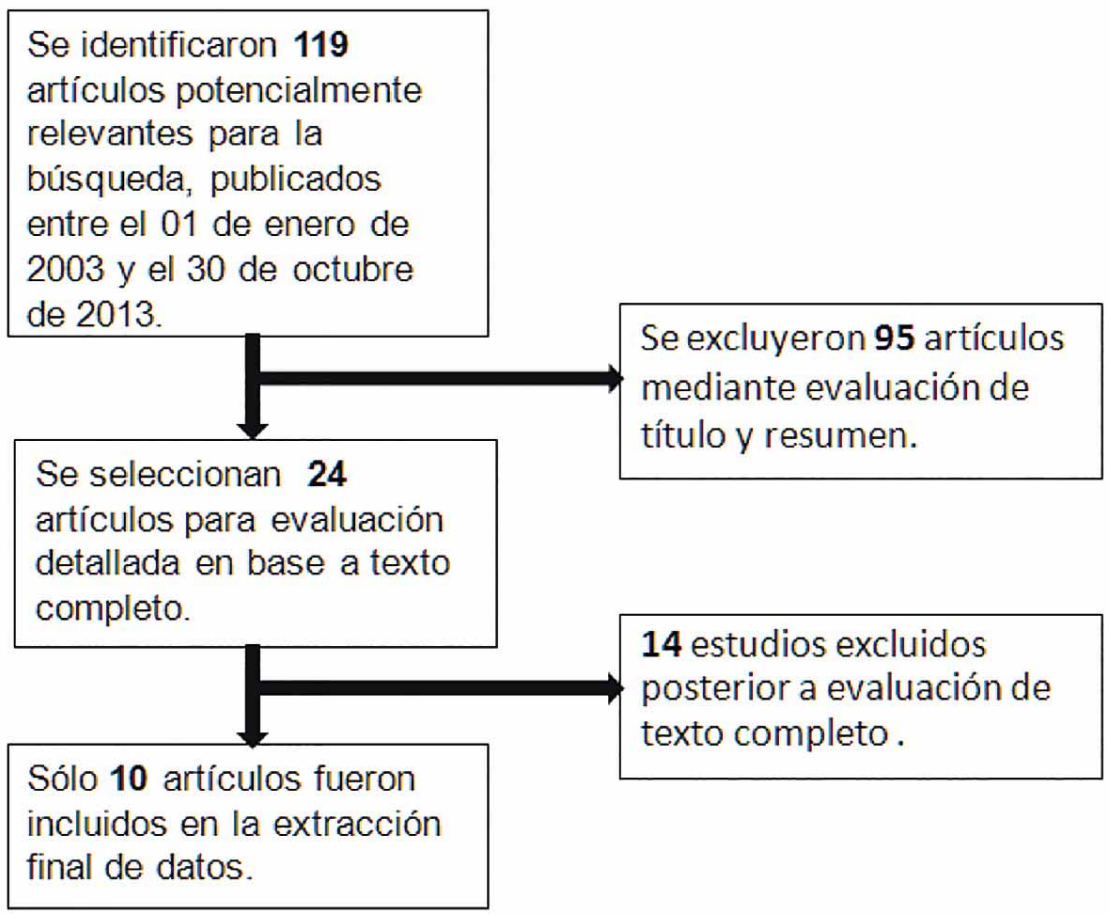

Fig. 1. Estrategia de búsqueda para estudiar el comportamiento clínico y las consideraciones de las PPRI. 
teriores no están protegidos por un tope dentario (Wills \& Manderson, 1977; Shahmiri et al., 2013). Esto conlleva en ocasiones a un desajuste de los retenedores, desarmonía oclusal y dolor en los tejidos blandos debajo del conector o de la base de la prótesis, que se observa con frecuencia después de un uso a largo plazo (Ohkubo et al., 2008). Además de la constante reabsorción del reborde alveolar por la presión continua de la base, requiriendo mantenciones, rebasado o sustitución de la prótesis (de Freitas et al.).

Como una posible solución a esta problemática clínica se propone desde hace aproximadamente 20 años, el uso de un implante colocado en la extensión distal de la base de la prótesis, permitiendo transformar una Clase I ó II de Kennedy en una clase III, con un costo relativamente accesible (Shahmiri \& Atieh, 2010).

\section{Ventajas y Desventajas de PPRI como Alternativa de Tratamiento en Extremo Libre Mandibular. La indicación de PPRI en mandíbula con extremo libre es en situaciones clínicas donde la inserción de múltiples implantes está restringida por la altura y características óseas desfavorables o por dificultades económicas que suponen otras modalidades de tratamiento. Siendo en general indicadas en desdentición de Clase I ó II de Kennedy, con inserción de un implante hacia la exten- sión distal del extremo libre (de Freitas et al.).}

Las ventajas del tratamiento con PPRI han sido reportadas desde 1993, con Keltjens et al., quien planteó que el uso de implantes en la extensión distal de una PPR otorgaría una oclusión más estable. El desplazamiento distal de la base se reduce, independientemente de la zona de apoyo de esta (Sato et al., 2013; de Freitas et al.), además permite disminuir la extensión de la base protésica, otorgando al paciente una mayor comodidad y satisfacción, por la mejora en las características biomecánicas de las tradicionales PPR. Mitrani et al. (2003) reportan escasa reabsorción ósea bajo la base protésica. A su vez se observa una mayor retención, estabilidad y mejor función en este tipo de prótesis (Mijiritsky \& Karas, 2004).

Existen aspectos que aún requieren ser estudiados referente a las características de los implantes en combinación con PPR, como son la mejor ubicación del implante en la extensión distal mandibular, su longitud ideal, sistemas de retención que minimicen la pérdida ósea alrededor del implante, entre otros parámetros que procuren una adecuada distribución de las fuerzas con mínimo estrés clínico (de Freitas et al.).
Diferentes estudios in vitro e in vivo se han realizado con la finalidad de dar respuesta a algunas de estas interrogantes. En este sentido, Cunha et al. (2008) determinaron que localizar el implante cerca del diente pilar favorece una mejor distribución de las tensiones en los dientes de soporte y tejidos blandos.

Pellizzer et al. (2010) estudiaron los sistemas de fijación más utilizados en PPRI, mediante modelos con diferentes pilares a los que se aplicaron fuerzas axiales y oblicuas, siendo analizadas por el modelo bidimensional de elementos finitos, los resultados mostraron una mejor distribución de la tensión en las estructuras de soporte mediante esta modalidad de tratamiento. Semejante a lo anterior, es lo reportado por Ohkubo et al. (2007), quienes reportaron menor presión sobre los tejidos blandos con PPRI.

Pronóstico Protésico y de los Implantes. La evidencia reportada respecto al pronóstico del tratamiento con PPRI es un tanto limitada y en ocasiones ambigua. Al respecto como se aprecia en la Tabla I, el tiempo de seguimiento descrito es en general bajo, sin embargo, Mitrani et al., describen un adecuado comportamiento clínico de las PPRI en los primeros 4,5 años, con escasa pérdida ósea estudiada a nivel radiográfico (0,1 $\mathrm{mm}$ por año posterior al primer año).

Entre las principales complicaciones que se observan destacan fracturas de la base protésica, aflojamiento de pilares de cicatrización e hiperplasia tisular (Payne et al., 2006), sin embargo, no existen datos estadísticos al respecto. Se ha señalado la necesidad de mantenciones periódicas del tratamiento protésico a fin de controlar su adecuado funcionamiento para corregir a tiempo los defectos que se susciten (de Freitas, et al.).

El pronóstico de los implantes es ampliamente informado y considera los criterios tradicionales de éxito clínico con tasas de supervivencia aceptables, de entre $93,7 \%$ y $100 \%$ (Tabla I). En la Tabla I, se expone un resumen de los ensayos clínicos encontrados para la búsqueda que se ajustaron a los criterios de selección.

\section{DISCUSIÓN}

La modalidad de tratamiento ofrecida por las PPRI es una rehabilitación relativamente económica y satisfactoria para el manejo de la desdentición par- 


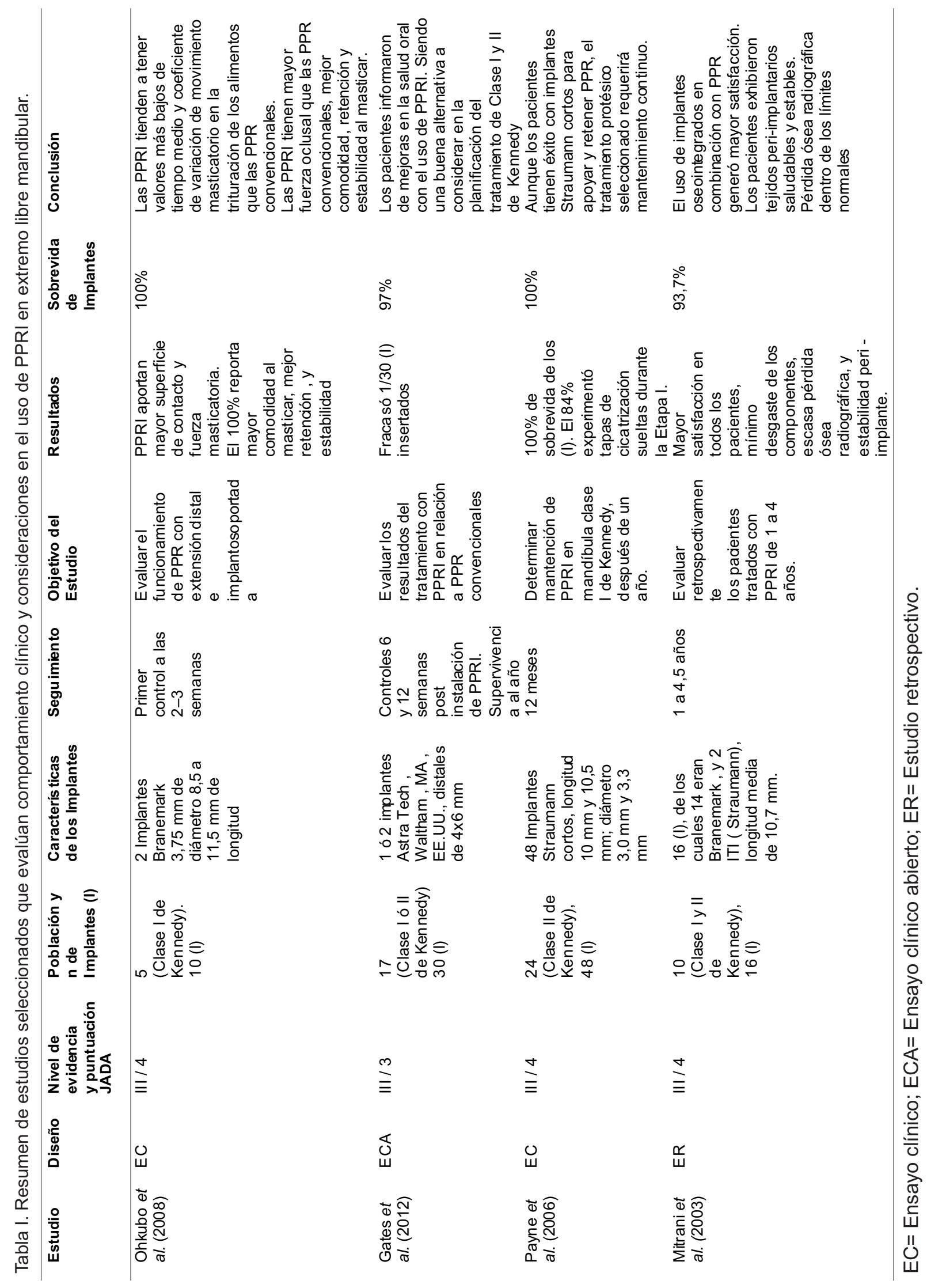


cial inferior. En esta investigación se estudiaron las consideraciones clínicas y el comportamiento de esta alternativa de tratamiento en la extensión distal mandibular.

La indicación de PPRI en mandíbula con extremo libre es en situaciones en que no es posible la inserción de múltiples implantes ya sea por condiciones óseas inadecuadas o por dificultades económicas, y por la mala experiencia previa con PPR convencionales (de Freitas et al.).

El respaldo de esta alternativa de tratamiento del extremo libre con la incorporación de un implante para el apoyo distal de la PPR convencional se basa en la mejora del comportamiento biomecánico de esta prótesis. Para comprobar dicho planteamiento, Pellizzer et al., llevaron a cabo un estudio in vitro y concluyó que la distribución de la tensión en las estructuras de soporte es mejor con la incorporación de un implante en la extensión distal mandibular, ésta distribución es mejor cuando la inclinación de los implantes es baja, es decir de entre $0^{\circ}$ y $5^{\circ}$ en sentido buco-lingual.

A su vez el uso de implantes en la extensión distal reduce la carga sobre la articulación temporomandibular, además al ser ubicados en la región del segundo molar, el centro de fuerza oclusal sería similar al de una prótesis fija implantosoportada (Bortolini et al., 2011).

En cuanto a la fuerza oclusal, Ohkubo et al. (2008) en un estudio in vivo determinaron mayor fuerza en las PPRI, lo que puede contribuir a una mejor capacidad de masticatoria debido a que a mayor fuerza oclusal mayor es el área de contacto.

Referente al comportamiento clínico se evidencia un aumento de la satisfacción del paciente al usar implantes en el extremo libre mandibular, otorgando mayor estabilidad a la prótesis (Ohkubo et al.; 2007; Gates et al., 2014), debido a mejoras en las propiedades biomecánicas de estas, al promover una mejor retención, con reducción del estrés en los dientes pilares, otorgando así mayor comodidad (de Freitas et al.). También, Ohkubo et al. (2007) reportaron in vitro que el apoyo del implante ayudaba a prevenir el desplazamiento de las prótesis y la disminución de la presión en los tejidos blandos. Sin embargo es escasa la literatura in vivo respecto a la reducción de la reabsorción ósea del reborde alveolar y sus efectos en tejidos adyacentes.

En relación a la supervivencia de implantes en extensiones distales de PPR mandibulares se reportan tasas favorables, con valores de entre $95 \%$ y $100 \%$, sin embargo, los períodos de seguimiento son limitados (Payne et al.; Ohkubo et al., 2008; Gates et al.). El estudio de mayor tiempo de seguimiento reportado es el presentado por Bortolini et al., con ocho años de evolución, presentando tasas de sobrevida del 93,75\%, sin embargo, ellos incluyeron en sus resultados el pronóstico de implantes insertados en el maxilar.

Las principales complicaciones informadas en esta modalidad de tratamiento son la continua necesidad de rebasado, desajuste de la unión prótesis-implante, daños en la base o acrílico de la prótesis (de Freitas et al.).

Entre las limitaciones de esta revisión destaca la diversidad de diseños metodológicos de la literatura publicada al respecto, siendo en general, de un bajo nivel de evidencia, principalmente casos o serie de casos clínicos, lo que impide un adecuado análisis de los datos, siendo necesarios estudios donde se elimine o minimice la confusión de ciertas variables para la comprensión de lo que ocurre en el manejo de extremo libre mandibular con PPRI para guiar al clínico a la mejor decisión terapéutica .

\section{CONCLUSIÓN}

Se incluyeron en este estudio revisiones sistemáticas y ensayos clínicos, por inexistencia de metaanálisis. A pesar de la diversidad metodológica observadas en los ensayos incluidos, las conclusiones observadas en la sobrevida de los implantes, satisfacción del paciente y características biomecánicas son similares.

El uso de PPR en combinación con implantes mejoraría las características biomecánicas de las tradicionales PPR, lo que se asocia a una mayor comodidad y bienestar para los pacientes desdentados de clase I y II de Kennedy, manteniendo la misma tasa de éxito de los implantes asociados a otras modalidades de tratamiento.

La utilización de un implante distal en combinación a una PPR de extremo libre pudiera ser una alternativa viable de tratamiento. Sin embargo, es necesario realizar estudios clinicos controlados, aleatorizados, a fin de estandarizar la técnica y los criterios de éxito protésico, para mejorar la seguridad, predictibilidad y consideraciones clínicas de una alternativa de tratamiento relativamente reciente. 
ARELLANO-VALERIA, M. J.; OLIVARES-KELLER, D. \& FLORES, M. A. Removable partial denture with distal extension and implant support, behavior and clinical consideration. Int. J. Odontostomat., 8(3):419-424, 2014.

ABSTRACT: The rehabilitation of free ends in a unilateral or bilateral jaw represents a major challenge for the dentist, and is a problem to solve in the daily clinical practice. These cases are usually resolved by removable partial dentures, which should be able to withstand horizontal forces. Torque adverse effect can occur during functioning, because of torque forces terminals against pillars and soft tissue mainly caused by the different resilience of these structures, therefore affecting retention, stability and support of the dental prosthesis. In recent times it has been reported that mandibular removable partial dentures in combination with distal free end implants can be a viable alternative to traditional removable partial dentures and implantsupported fixed partial dentures for treatment of partial toothless free end, however literature regarding functionality and prognosis of this alternative is limited. This paper presents a systematic literature review aimed to identify and synthesize the best available evidence regarding the considerations and clinical behavior of this treatment modality in edentulous Class I and II of the edentulous Kennedy mandibular.

KEY WORDS: removable partial denture implant, removable partial dentures on implants, free end.

\section{REFERENCIAS BIBLIOGRÁFICAS}

Bortolini, S.; Natali, A.; Franchi, M.; Coggiola, A. \& Consolo, U. Implant-retained removable partial dentures: an 8-year retrospective study. J. Prosthodont., 20(3):168-72, 2011.

Budtz-Jorgensen, E. Prosthodontics for the Eldery: Diagnosis and Treatment. Chicago, Quintessence Publishing Co. Inc., 1999.

Cunha, L. D.; Pellizzer, E. P.; Verri, F. R. \& Pereira, J. A. Evaluation of the influence of location of osseointegrated implants associated with mandibular removable partial dentures. Implant Dent., 17(3):278-87, 2008.

de Freitas, R. F.; se Carvalho Dias, K.; da Fonte Porto Carreiro, A.; Barbosa, G. A. \& Ferreira, M. A. Mandibular implantsupported removable partial denture with distal extension: a systematic review. J. Oral Rehabil., 39(10):791-8, 2012.

Gates, W. D. 3rd.; Cooper, L. F.; Sanders, A. E.; Reside, G. J. \& De Kok, I. J. The effect of implant-supported removable partial dentures on oral health quality of life. Clin. Oral Impl. Res., 25(2):207-13, 2014.

Mijiritsky, E. \& Karas, S. Removable partial denture design involving teeth and implants as an alternative to unsuccessful fixed implant therapy: a case report. Implant Dent., 13(3):21822, 2004.

Mitrani, R.; Brudvik, J. S. \& Philips, K. M. Posterior implants for distal extension removable prostheses: a retrospective study. Int. J. Periodontics Restorative Dent., 23(4):353-9, 2003.

Ohkubo, C.; Kobayashi, M.; Suzuki, Y. \& Hosoi T. Effect of implant support on distal-extension removable partial dentures: in vivo assessment. Int. J. Oral Maxillofac. Implants, 23(6):1095-101, 2008.

Ohkubo, C.; Kurihara, D.; Shimpo, H.; Suzuki, Y.; Kokubo, Y. \& Hosoi, T. Effect of implant support on distal extension removable partial dentures: in vitro assessment. J. Oral Rehabil., 34(1):52-6, 2007.
Payne, A.; Kuzmanovic, D. V.; De Silav-Kumara, R. \& van Staden, I. P. Mandibular removable partial dentures supported by implants: one-year prosthodontics outcomes. J. Dent. Res., 85:2570, 2006.

Pellizzer, E. P.; Verri, F. R.; Falcón-Antenucci, R. M.; Goiato, M. C. \& Gennari Filho, H. Evaluation of different retention systems on a distal extension removable partial denture associated with an osseointegrated implant. J. Craniofac. Surg., 21(3):727-34, 2010.

Sato, M.; Suzuki, Y.; Kurihara, D.; Shimpo, H. \& Ohkubo, C. Effect of implant support on mandibular distal extension removable partial dentures: relationship between denture supporting area and stress distribution. J. Prosthodont. Res., 57(2):109-12, 2013.

Shahmiri, R.; Aarts, J. M.; Bennani, V.; Das R. \& Swain, M. V. Strain Distribution in a Kennedy Class I Implant Assisted Removable Partial Denture under Various Loading Conditions. Int. J. Dent., 2013:351279, 2013.

Shahmiri, R. A. \& Atieh, M. A. Mandibular Kennedy Class I implanttooth-borne removable partial denture: a systematic review. J. Oral Rehabil., 37(3):225-34, 2010.

Turkyilmaz, I. Use of distal implants to support and increase retention of a removable partial denture: a case report. $J$. Can. Dent. Assoc., 75(9):655-8, 2009.

Wills, D. J. \& Manderson, R. D. Biomechanical aspects of the support of partial dentures. J. Dent., 5(4):310-8, 1977.

Dirección para correspondencia:

Prof. Dr. Marco A. Flores V.

Escuela de Odontología

Facultad de Odontología

Universidad de La Frontera

Claro Solar 115

Temuco- CHILE

Recibido : 29-01-2014

Aceptado: 09-09-2014

Email: marco.flores@ufrontera.cl 